\title{
Locality and Unitarity of Scattering Amplitudes from Singularities and Gauge Invariance
}

\author{
Nima Arkani-Hamed, ${ }^{1}$ Laurentiu Rodina, ${ }^{2}$ and Jaroslav Trnka ${ }^{3}$ \\ ${ }^{1}$ School of Natural Sciences, Institute for Advanced Study, Princeton, New Jersey 08540, USA \\ ${ }^{2}$ Department of Physics, Princeton University, Jadwin Hall, Princeton, New Jersey 08540, USA \\ ${ }^{3}$ Center for Quantum Mathematics and Physics (QMAP), Department of Physics, \\ University of California, Davis, California 95616, USA
}

(Received 23 December 2017; revised manuscript received 9 May 2018; published 8 June 2018)

\begin{abstract}
We conjecture that the leading two-derivative tree-level amplitudes for gluons and gravitons can be derived from gauge invariance together with mild assumptions on their singularity structure. Assuming locality (that the singularities are associated with the poles of cubic graphs), we prove that gauge invariance in just $n-1$ particles together with minimal power counting uniquely fixes the amplitude. Unitarity in the form of factorization then follows from locality and gauge invariance. We also give evidence for a stronger conjecture: assuming only that singularities occur when the sum of a subset of external momenta go on shell, we show in nontrivial examples that gauge invariance and power counting demand a graph structure for singularities. Thus, both locality and unitarity emerge from singularities and gauge invariance. Similar statements hold for theories of Goldstone bosons like the nonlinear sigma model and Dirac-Born-Infeld by replacing the condition of gauge invariance with an appropriate degree of vanishing in soft limits.
\end{abstract}

DOI: 10.1103/PhysRevLett.120.231602

Gauge redundancy.-The importance of gauge invariance in our description of physics can hardly be overstated, but the fundamental status of "gauge symmetry" has evolved considerably over the decades. While many older textbooks rhapsodize about the beauty of gauge symmetry and wax eloquent on how "it fully determines interactions from symmetry principles", from a modern point of view gauge invariance can also be thought of as by itself an empty statement. Indeed any theory can be made gauge invariant by the "Stuckelberg trick"-elevating gaugetransformation parameters to fields-with the "special" gauge invariant theories distinguished only by realizing the gauge symmetry with the fewest number of degrees of freedom.

Instead of gauge symmetry, we speak of gauge "redundancy" as a convenient but not necessarily fundamental way of describing the local physics of Yang-Mills and gravity theories. From the modern point of view, gauge symmetry is merely useful for making locality and unitarity manifest when describing the physics of an interacting massless particle of spin one or two.

Over the past few decades, we have seen entirely different formalisms for computing scattering amplitudes not tied to this formalism, and here, gauge redundancy

Published by the American Physical Society under the terms of the Creative Commons Attribution 4.0 International license. Further distribution of this work must maintain attribution to the author(s) and the published article's title, journal citation, and DOI. Funded by SCOAP ${ }^{3}$. makes no appearance whatsoever. Instead of polarization vectors that only redundantly describe massless particle states, we can use spinor-helicity variables, which trivially incorporate gauge invariance.

With this description of amplitudes at hand, it becomes possible to pursue entirely new strategies for determining the amplitudes. In a first stage, one can speak of a modern incarnation of the $S$-matrix program, where the fundamental physics of locality and unitarity are imposed to determine the amplitudes from first principles. This has allowed the computation of amplitudes in an enormous range of theories, from Yang-Mills and gravity to Goldstone bosons, revealing stunning simplicity and deep new mathematical structures that are completely hidden in the usual, gauge-redundant Feynman diagram formalism. Conversely and more ambitiously, these developments suggest that what we think of as "scattering amplitudes from local evolution in spacetime" might fundamentally be something entirely different: instead of merely exploiting locality and unitarity to determine the amplitudes, we seek "scattering amplitudes" as the answer to very different natural mathematical questions, and only later discover that the results are local and unitary. Carrying this program out in full generality for all interesting theories would likely shed powerful new light on a deeper origin for both spacetime and quantum mechanics itself.

A step in this direction has been taken with the discovery of the "amplituhedron" [1], a geometric object generalizing plane polygons to higher-dimensional spaces, whose "volume" computes scattering amplitudes for maximally supersymmetric four-dimensional theories in the planar 
limit (in particular, giving tree-level gluon scattering amplitudes for the real theory of strong interactions relevant for particle collisions at the LHC). In this example, we can see concretely how the usual rules of spacetime and quantum mechanics emerge from more primitive principles.

Role reversal.- In this Letter, we explore aspects of locality and unitarity from a point of view entirely orthogonal to these recent developments. As emphasized above, much of the explosion of progress in understanding scattering amplitudes has taken place precisely by eschewing any reference to gauge redundancy and working directly with the physical on-shell amplitudes. Here, we instead return to the requirement of on-shell gauge invariance as primary and consider rational functions built out of polarization vectors and momenta, without making any reference to underlying Lagrangian or Feynman rules. Surprisingly, we find that with mild restrictions on the form of functions we consider the requirement of on-shell gauge invariance uniquely fixes the functions to match the tree amplitudes of Yang-Mills theory for spin one and gravity for spin two. There is a similar story determining the amplitudes for Goldstone bosons of the nonlinear sigma model and the Dirac-Born-Infeld action, where the requirement of on-shell gauge invariance is replaced by an appropriate vanishing of amplitudes in soft limits.

Suppose that we are handed a rational function of momenta and polarization vectors. What constraints determine this function to correspond to "scattering amplitudes"? One might imagine that both locality and unitarity are crucially needed for this purpose. In other words, we have to assume that this function has only simple poles when the sum of a subset $\mathcal{S}$ of the momenta $P_{\mathcal{S}}^{\mu}=\sum_{i \subset \mathcal{S}} p_{i}^{\mu}$ goes on shell; i.e., the only singularities present look like $\sim 1 / P_{\mathcal{S}}^{2}$, and the function factorizes on the poles into the product of lower-point objects, with an extra intermediate line.

Our central claim is that while locality and unitarity must be imposed to determine amplitudes for garden-variety scalar theories like $\phi^{3}$, mисh less is needed to uniquely fix the function to be "the amplitude" for gauge theories and gravity. In fact, we conjecture that simply specifying that singularities only occur when the sum of a subset of momenta goes on shell $P^{2} \rightarrow 0$, together with the usual mass dimension power counting (which also enforces nontrivial gauge invariance) uniquely fixes the function. We sketch the essential ideas in this Letter; a more detailed exposition of our proof and other related results appear in [2]. Other observations about the surprisingly restrictive power of on-shell gauge invariance have recently been made in [3].

To begin, we can enforce only locality and look at functions whose singularities are propagator poles appearing in cubic graphs. But we do not demand unitarity: we do not demand that the function factorizes on the poles. We find that the leading nontrivial gauge invariants with the singularities of cubic graphs are unique and give us amplitudes. The necessity of factorization-and thus unitarity-follows from locality and gauge invariance. We sketch a straightforward proof of this fact, which begins by showing that given the poles of cubic graphs gauge invariance alone (with no assumption about factorization) fixes the structure of the soft limit of any expressions to reproduce the usual Weinberg soft theorems [4].

But we also make a stronger conjecture that even the structure of singularities associated with cubic graphs need not be enforced: we need only assume that the singularities occur when $P_{\mathcal{S}}^{2} \rightarrow 0$. We consider functions that have at most degree $\beta$ singularities of this form, and that is our most general ansatz is

$$
\sum_{\left\{\mathcal{S}_{1}, \ldots, \mathcal{S}_{\beta}\right\}} \frac{N_{i}\left(p^{\alpha}\right)}{P_{\mathcal{S}_{1}}^{2} \cdots P_{\mathcal{S}_{\beta}}^{2}} .
$$

Here, $N_{i}\left(p^{\alpha}\right)$ is a polynomial in the momenta (and linear in all the polarization vectors), with a total of $\alpha$ momenta.

We now only ask for this expression to be on-shell gauge invariant. The answer is unique, if we limit to the lowest possible value for $\alpha$, which turns out to be $\alpha=n-2$. Similarly, the lowest number of singularities needed to find a solution is $\beta=n-3$.

This unique object picks out the singularities from cubic graphs and factorizes on poles; locality and unitarity arise from singularities and gauge invariance.

All of these statements are made in general $D$ spacetime dimensions: we are working with Lorentz invariants of the form $\left(\epsilon_{i} \cdot \epsilon_{j}\right),\left(\epsilon_{i} \cdot p_{j}\right)$, and $\left(p_{i} \cdot p_{j}\right)$, satisfying the relations $p_{i}^{2}=0, \epsilon_{i} \cdot p_{i}=0$, and momentum conservation $\sum_{i} p_{i}^{\mu}=0$.

Locality, unitarity, and gauge invariance.-Let us begin by focusing on the tree-level scattering amplitudes in Yang-Mills theory; we later summarize the precisely analogous statements for gravity. The group structure of gluon amplitudes can be stripped off in trace factors $\mathcal{A}_{n}=\sum_{\sigma / Z} \operatorname{Tr}\left(T^{\sigma_{1}} T^{\sigma_{2}} \ldots T^{\sigma_{n}}\right) A_{n}(123 \ldots n)$, where $A_{n}$ is an ordered amplitude which is a gauge invariant cyclic object. All poles in $A_{n}$ are local cyclic factors, $P_{i j}^{2}=$ $\left(p_{i}+p_{i+1}+\cdots p_{j}\right)^{2}$. On these poles, $A_{n}$ factorizes as a product of two ordered amplitudes,

$$
\lim _{P^{2} \rightarrow 0} A_{n}=\sum_{h} A_{L}^{\left(h_{L}\right)} \frac{1}{P^{2}} A_{R}^{\left(h_{R}\right)}
$$

where we sum over all internal degrees of freedom $h$.

The cyclic amplitude $A_{n}$ can be calculated using colorordered Feynman rules. For each cubic graph $\Gamma$, we get

$$
D_{n}^{(\Gamma)}=\frac{N_{n}^{(\Gamma)}\left(\epsilon_{i}, p_{j}\right)}{P_{\sigma_{1}}^{2} P_{\sigma_{2}}^{2} \ldots P_{\sigma_{n-3}}^{2}},
$$

where all the factors $P_{\sigma_{a}}^{2}$ in the denominator come from Feynman propagators of cubic diagrams. The numerator is 
a polynomial in all polarization vectors $\epsilon_{i}$ and $n-2$ momenta $p_{j}$. For the diagrams with four point vertices, we get fewer than $n-3$ propagators, but they can be also put (nonuniquely) in the form by multiplying both numerator and denominator by some $P^{2}$.

Feynman diagrams are designed to make locality and unitarity manifest, but gauge invariance is not manifest diagram-by-diagram: we have to sum over all Feynman diagrams to get a gauge invariant expression. The tension between locality, unitarity, and gauge invariance is vividly seen in the four-particle amplitude. The color ordered amplitude $A_{4}$ is a sum of three Feynman diagrams, schematically written as (ignoring all indices)

$$
A_{4} \sim \frac{(\epsilon \cdot p)(\epsilon \cdot \epsilon)}{s}+\frac{(\epsilon \cdot p)(\epsilon \cdot \epsilon)}{t}+(\epsilon \cdot \epsilon)(\epsilon \cdot \epsilon) .
$$

Only the sum of all three terms is gauge invariant which can be made manifest once we write $A_{4}$ as

$$
A_{4} \sim \frac{F^{4}}{s t},
$$

where the numerator is just a (color-ordered) local amplitude. This expression is trivially gauge invariant, but we do not have manifest locality and unitarity.

Unitarity from locality and gauge invariance.-First, we would like to build an ansatz compatible with locality. Consider the set of all cubic graphs with cyclic ordering of external legs and for each of them write an expression $\tilde{D}_{n}^{(\Gamma)}$ of the form (3) where the poles in the denominator are dictated by the internal lines of the given graph. Unlike in Feynman diagrams, we do not demand the numerator comes from Feynman rules, and therefore, we are not imposing unitarity or factorization. Instead, we take $N_{n}^{(\Gamma)}\left(p^{n-2}\right)$ to be an arbitrary polynomial of degree $n-2$ in momenta $p_{j}$ and $n$ polarization vectors $\epsilon_{i}$.

Now, we now consider a sum of all expressions associated with graphs $\Gamma$,

$$
\tilde{A}_{n}\left(p^{n-2}\right)=\sum_{\Gamma} \tilde{D}_{n}^{(\Gamma)}\left(p^{n-2}\right),
$$

and impose gauge invariance in just $n-1$ legs. We claim that this specifies a unique expression which is an $n$ point tree-level amplitude $\tilde{A}_{n}=A_{n}$.

The proof goes as follows: we assume inductively that $\tilde{A}_{n}=A_{n}$ is unique for the $n$ particle case and that there is no local tensor $B_{n}^{\mu_{1} \ldots \mu_{m}}\left(p^{k}\right)$ gauge invariant in $k+1$ particles for $k \leq n-2$. Now, we take the expansion (6) for $n+1$ particles and go to the soft limit $p_{n+1} \equiv q \rightarrow 0$. It is easy to show that gauge invariance requires the leading divergent term to be a Weinberg soft factor,

$$
\tilde{A}_{n+1}=\left(\frac{\epsilon \cdot p_{1}}{q \cdot p_{1}}-\frac{\epsilon \cdot p_{n}}{q \cdot p_{n}}\right) B_{n}\left(p^{n-2}\right)+\mathcal{O}(1),
$$

where $B_{n}$ is the gauge invariant function in $n-1$ legs with $n-2$ powers of momenta, so it must be equal to $A_{n}$ by induction. Now, consider instead the object $M_{n+1}=$ $\tilde{A}_{n+1}-A_{n+1}$, which has a vanishing leading piece. This is important because a nonzero order $\mathcal{O}\left(z^{m}\right)$ has a contribution to order $\mathcal{O}\left(z^{m+1}\right)$ through momentum conservation (see [5] for a discussion). Then, the subleading piece in the soft limit has the form,

$$
\delta_{1} M_{n+1}=\frac{\epsilon^{\mu} q^{\nu} B_{n}^{\mu \nu}\left(p^{n-2}\right)}{q \cdot p_{1}}+\frac{\epsilon^{\mu} q^{\nu} \bar{B}_{n}^{\mu \nu}\left(p^{n-2}\right)}{q \cdot p_{n}},
$$

where we omitted the terms with double poles which are directly ruled out by gauge invariance. The tensors $B_{n}^{\mu \nu}, \bar{B}_{n}^{\mu \nu}$ have $k=n-2$, and so, our second assumption rules them out. At higher orders in the soft limit, we always get $\delta_{p} M_{n+1} \sim X_{n}^{\mu \nu}\left(p^{k}\right)$ for some tensor $X$, which is then ruled out, and all these terms must vanish. The second assumption itself can be proven by an identical inductive reasoning.

It is interesting that in these arguments it suffices to check gauge invariance only in $n-1$ legs to uniquely fix the answer. This observation explains why the object factorizes on poles. We would like to determine what our unique gauge invariant looks like on a factorization channel. Since there is already a unique gauge invariant only checking invariance on $n-1$ legs, we can take "left" and "right" gauge invariants ignoring gauge invariance on the intermediate line; gluing together these unique objects then gives us something that is gauge invariant in all $n$ legs and therefore must match the unique $n$ point gauge invariant on this channel. This shows that gauge invariants factorize on poles, allowing us to see the emergence of unitarity very directly.

Locality from gauge invariance.-We showed that unitarity is a derived property of gluon amplitudes if we demand only locality and gauge invariance. But we can go even further and even remove the requirement of locality. We again consider a sum of terms (6), but now, we give up on the assumption that individual terms (3) have poles which correspond to cubic diagrams. We just consider any cyclic poles $P_{i j}^{2}=\left(p_{i}+p_{i+1}+\cdots+p_{j}\right)^{2}$ and even allow powers $\left(P_{i j}^{2}\right)^{\#}$. The only assumption is that the total number of poles in the denominator is $n-3$. For example, for the $n=5$ case, we allow terms of the form

$$
\frac{N_{5}^{(1)}}{s_{12}^{2}}, \quad \frac{N_{5}^{(2)}}{s_{12} s_{23}} .
$$

While the double (or higher) poles can come from the Lagrangians with a noncanonical kinetic term, the second term cannot be associated with any local interaction as it does not correspond to any "diagram" of particle scattering.

We now conjecture that if we impose gauge invariance on the general sum of all possible terms with $n-3$ cyclic 
poles (6), the only solution is the $n$ point scattering amplitude $A_{n}$. We have directly checked this conjecture by brute force up to $n=5$, which is already highly nontrivial.

Gravity and BCJ.-The story for gravitons is essentially identical. We consider cubic graphs now with no ordering of external legs, and for each graph, we associate an expression (3). The numerator $N_{n}^{(\Gamma)}$ is a polynomial of degree $2(n-2)$ in momenta $p_{i}$, and it also depends on $n$ polarization tensors $\epsilon_{\mu \nu}=\epsilon_{\mu} \epsilon_{\nu}$.

Imposing the gauge invariance condition in $n-1$ external legs we get a unique solution: the graviton amplitude. Therefore, unitarity emerges from locality and gauge invariance like for Yang-Mills theory, and the proof follows identical steps. The stronger statement also holds in this case: even without assuming a cubic structure, the gravity amplitude is the unique gauge invariant object.

We can also go back to the gluon case and consider all possible $P^{2}$ poles, not just the ones with cyclic momenta, but now choosing $n-3$ of all possible cubic graph poles. Imposing gauge invariance, we conjecture $(n-2)$ ! solutions corresponding to different cyclic orderings of YangMills amplitudes, modulo the relations following from the $U(1)$ decoupling and KK relations.

The uniqueness of gauge invariants also gives a natural proof for the BCJ relation [6] between the Yang-Mills and gravity amplitudes. If we write the Yang-Mills amplitude in the BCJ form, such that the kinematical numerators satisfy the same Jacobi identities as the corresponding color factors, then the gravity amplitude is given by the simple replacement of the color factor by one more power of the kinematical numerator,

$$
A_{n}^{(Y M)}=\sum_{\Gamma} \frac{N_{\Gamma} c_{\Gamma}}{D_{\Gamma}} \rightarrow A_{n}^{(G R)}=\sum_{\Gamma} \frac{N_{\Gamma}^{2}}{D_{\Gamma}} .
$$

The reason is very simple. Under a gauge variation, the $N_{\Gamma}$ change by some $\Delta_{\Gamma}$; the invariance of the full amplitude $\sum_{\Gamma} c_{\Gamma} \Delta_{\Gamma} / D_{\Gamma}=0$ can then only be ensured by the Jacobi relations satisfied by $c_{\Gamma}$. But if we now replace $c_{\Gamma}$ with some kinematical factor $N_{\Gamma}$ which satisfies the same identities, the gravity-gauge invariance check follows in exactly the same way as for YM. Thus, the object with $c_{\Gamma} \rightarrow N_{\Gamma}$ is a gravitational gauge invariant with $2(n-2)$ powers of momenta in the numerator; since this object is unique, it gives the gravity amplitude.

Gauge invariance $\rightarrow$ soft limits and Goldstone theories.-Because of gauge invariance, we have seen that Yang-Mills and gravity amplitudes are much more special than garden-variety scalar theories. But recent investigations revisiting some classic aspects of Goldstone scattering amplitudes have revealed a similarly special property of scalar theories. In the case of the nonlinear sigma model, soft limit behavior in the form of the Adler zero [7] supplements unitarity and locality in certain cases to completely fix the tree-level $S$ matrix [8-10]. In general, we can ask what are the minimally derivatively coupled theories whose amplitudes have a vanishing soft limit $A_{n}=0$ for $p_{j} \rightarrow 0$ ? For linear vanishing, the answer appears to be nonlinear sigma model (NLSM). If we demand a quadratic vanishing $A_{n}=\mathcal{O}\left(p^{2}\right)$, this uniquely specifies the Dirac-Born-Infeld (DBI) theory and $A_{n}=\mathcal{O}\left(p^{3}\right)$ gives a special Galileon $[8,11]$. The soft limit behavior was then used in the recursion relations to reconstruct the amplitudes in these theories, supplementing locality and unitarity.

In the spirit of our previous statements, we can make similar claims for these theories. Like for Yang-Mills, we can strip the flavor factor in the NLSM amplitude[12] and consider cyclically ordered amplitudes $A_{n}$. Now, the individual Feynman diagrams are quartic diagrams $\mathcal{Q}$, and we can write an expression for each,

$$
D_{n}^{(\mathcal{Q})}=\frac{N_{n}^{(\mathcal{Q})}\left(p^{n-2}\right)}{P_{1}^{2} P_{2}^{2} \ldots P_{n / 2-2}^{2}} .
$$

Imposing the soft limit vanishing then requires summing over all Feynman diagrams as only the amplitude has this property. Now, we consider a general numerator,

$$
N_{n}^{(\mathcal{Q})}\left(p^{n-2}\right)=\sum_{k} \alpha_{k} \Delta_{k},
$$

where $\Delta_{k}$ is the product of $n / 2-1$ terms of the form $s_{i j}=\left(p_{i} \cdot p_{j}\right)$.

We claim that imposing the soft limit vanishing in $n-1$ legs fixes all coefficients completely, leaving a unique expression, the $n$ point amplitude in the NLSM. The proof for this statement uses a double soft limit in which two of the momenta go to zero. In that case, the amplitude does not vanish but rather gives a finite expression, the analogue of the Weinberg soft factor. One can then prove the statement in a similar way to the soft limit argument for gluons and gravitons.

The stronger claim is that we do not have to consider quartic graphs but rather take any expression with $n / 2-2$ factors in the denominator. Then imposing the soft limit still fixes the result uniquely, and we can see both locality and unitarity arising from the vanishing in the soft limit.

We can make analogous claims for the DBI and special Galileon. The power counting of the numerators is $n-2$, respectively $3 n / 2-3$, factors $s_{i j}$. We have to consider all quartic graphs with no ordering. Imposing the $\mathcal{O}\left(p^{2}\right)$, respectively $\mathcal{O}\left(p^{3}\right)$, vanishing in the soft limit of $n-1$ legs we get the corresponding amplitude as the unique solution. The stronger statement again removes the requirement of poles associated with quartic diagrams.

Outlook.-There are a number avenues for further exploration suggested by this work. First, all of our analyses find objects that would be gauge invariant in any number of 
dimensions. But in a specific spacetime dimensionality $d$, there are further "gram determinant" conditions arising from the linear interdependence of momenta, which could, in principle, affect the uniqueness results.

Provided that the absence of dimension-specific invariants can be established, we have found a simple conceptual understanding of a fact that has resisted a transparent understanding for many years. There is an apparently straightforward proof that "amplitudes that factorize properly" must match Feynman diagrams, by using Cauchy's theorem and the BCFW deformation [13] to show that if functions have the same singularities they must be equal. However, this famously needs a proof of an absence of poles at infinity on the Feynman diagram side, which can only be shown by a relatively indirect argument far afield from on-shell physics [14].

The uniqueness of gauge invariance implies further properties of the $S$ matrices. In particular, it is trivial to show that it is impossible to have interactions of higher spin particles. The standard modern $S$-matrix argument relies on the factorization of the four point amplitude which is inconsistent in all three channels for $s>2[15,16]$. In our story, we do not use factorization. Gauge invariance alone implies that any amplitude with cubic graphs needs a Weinberg soft factor, and that is impossible to construct for higher spins.

Our results also illuminate why the CHY construction [17] of YM and gravity amplitudes must match the correct answer, without any detailed analysis of the poles and factorization structure. We simply observe that the poles of the $\mathrm{CHY}$ formula are local, and the expressions are gauge invariant expressions, with the correct units to match the correct numerator power counting.

Finally, while the claims in this Letter are mathematically nontrivial and certainly have physical content, their ultimate physical significance is not clear. It is intriguing that locality and unitarity can be derived from the redundancy, inverting the usual logic leading to the need for gauge invariance. If this is more than a curiosity, it would be interesting to look for an abstract underlying system that gives rise to an effective description-either exactly or approximately - with a gauge redundancy from which locality and unitarity emerge in the way we have seen here.

We thank Freddy Cachazo, John Joseph Carrasco, David Kosower, and Rutger Boels for stimulating discussions. The work of N.A.H. is supported in part by the U.S. Department of Energy under Grant No. DOE DESC0009988. J. T. is supported by a DOE Grant No. DESC0009999.

[1] N. Arkani-Hamed and J. Trnka, J. High Energy Phys. 10 (2014) 030.

[2] L. Rodina, arXiv:1612.03885; arXiv:1612.06342.

[3] R. H. Boels and R. Medina, Phys. Rev. Lett. 118, 061602 (2017).

[4] S. Weinberg, Phys. Rev. 140, B516 (1965).

[5] J. Broedel, M. de Leeuw, J. Plefka, and M. Rosso, Phys. Rev. D 90, 065024 (2014).

[6] Z. Bern, J. J. M. Carrasco, and H. Johansson, Phys. Rev. D 78, 085011 (2008).

[7] S. L. Adler, Phys. Rev. 137, B1022 (1965).

[8] C. Cheung, K. Kampf, J. Novotny, and J. Trnka, Phys. Rev. Lett. 114, 221602 (2015).

[9] C. Cheung, K. Kampf, J. Novotny, C. H. Shen, and J. Trnka, Phys. Rev. Lett. 116, 041601 (2016).

[10] C. Cheung, K. Kampf, J. Novotny, C. H. Shen, and J. Trnka, J. High Energy Phys. 02 (2017) 020.

[11] F. Cachazo, S. He, and E. Y. Yuan, J. High Energy Phys. 07 (2015) 149.

[12] K. Kampf, J. Novotny, and J. Trnka, J. High Energy Phys. 05 (2013) 032.

[13] R. Britto, F. Cachazo, B. Feng, and E. Witten, Phys. Rev. Lett. 94, 181602 (2005).

[14] N. Arkani-Hamed and J. Kaplan, J. High Energy Phys. 04 (2008) 076.

[15] P. Benincasa and F. Cachazo, arXiv:0705.4305.

[16] D. A. McGady and L. Rodina, Phys. Rev. D 90, 084048 (2014).

[17] F. Cachazo, S. He, and E. Y. Yuan, Phys. Rev. Lett. 113, 171601 (2014). 